Irish Math. Soc. Bulletin

Number 83, Summer 2019, 9-16

ISSN 0791-5578

\title{
The Hornich space and a subspace of BMOA
}

\author{
JOSEPH A. CIMA
}

\begin{abstract}
In this article we make a linear association between the Hornich space and a subspace of the BMOA space on the unit disc. We obtain a few new results that arise from the association and identify some classes of functions in BMOA that were hitherto unknown (or utilized).
\end{abstract}

\section{INTRODUCTION}

In this article we consider two normed spaces of analytic functions on the unit disc $D$ and produce a linear isomorphism between them. The purpose is not just to make the identification: it suggests a large class of functions that were hitherto unknown in one of the spaces. The first space, which we denote by $\mathscr{H}$, is a real Banach space and is known to those specialists who work in geometric function theory. The second is a certain subspace $\mathscr{K}$ of the space BMO of functions of bounded mean oscillation. Indeed, $\mathscr{K}$ is a subspace of the space BMOA of analytic functions of bounded mean oscillation. The space BMO has broader applications and is found in research in the areas of partial differential equations, harmonic analysis and other areas. There are classes of functions in $\mathscr{H}$ described by their geometry and it is of interest to see what the implications of the connection to the subspace of BMOA will imply about these subsets, and on the obverse side of the relation it will be of interest to see what the geometry will imply about the behavior of the associated BMOA functions.

\section{The Hornich Space And some of its PRoperties}

Let $\mathcal{A}$ denote the set of functions $f$ which are holomorphic on the unit $\operatorname{disc} D$, and which are locally univalent there (i.e. $f^{\prime}(z) \neq 0,|z|<1$ ), and normalized by $f(0)=0, f^{\prime}(0)=1$. For each $r \in(0,1)$ and $f \in \mathcal{A}$ define the quantity

$$
I(r ; f) \equiv \frac{1}{2 \pi} \int_{0}^{2 \pi}|\log | f^{\prime}\left(r e^{\imath t}\right)|| d t .
$$

We define a set $\mathscr{L}$ as follows.

$$
\mathscr{L}=\left\{f \in \mathcal{A} \mid \sup _{r}(I(r ; f))<\infty\right) .
$$

We define a few other sets which we wish to refer to later in this paper. Namely, set

$$
\mathscr{M}=\left\{\log f^{\prime}(z) \mid f \in \mathscr{L}\right\},
$$

and

$$
\mathscr{N}=\left\{f^{\prime} \mid f \in \mathscr{L}\right\} .
$$

2010 Mathematics Subject Classification. 30E99.

Key words and phrases. Analytic function, bounded mean oscillation, bounded arguments. Received on 15-4-2019. 
The set $\mathscr{M}$ is a subset of the space of Cauchy Transforms of finite Borel measures on the unit circle (see Cima and Pfaltzgraff [4] and Cima, Matheson, and Ross [3]). In particular there is a function $M(\zeta)$ of bounded variation on the unit circle $\mathbb{T}$ such that

$$
\log f^{\prime}(z)=\int_{\mathbb{T}} \frac{(\zeta+z)}{(\zeta-z)} d M(\zeta), \quad z \in D .
$$

Note that it is easy to see that each functions $f$ in $\mathscr{N}$ has derivative $f^{\prime}$ in the Nevanlinna class, and hence has radial boundary values $f^{\prime}(\zeta)$ at almost all $\zeta \in \mathbb{T}$.

For $f$ and $g$ in $\mathscr{L}$ define

$$
f \oplus g(z)=\int_{o}^{z} f^{\prime}(w) g^{\prime}(w) d w
$$

and for $r \in \mathbb{R}$ set

$$
r \odot f(z)=\int_{o}^{z}\left(f^{\prime}(w)\right)^{r} d w .
$$

With these operations, $\mathscr{L}$ becomes a real vector space, and the expression

$$
\|f\|_{\mathscr{L}} \equiv \sup _{0<r<1}(I(r ; f))
$$

is a norm on $\mathscr{L}$, and in this norm the space is complete, that is, $\mathscr{L}$ is a real Banach space. Although not much is known about a generic vector in $\mathscr{L}$ or the relationship of the entire space $\mathscr{L}$ to other classical Banach spaces of analytic functions (e.g. weighted Bergman spaces or weighted Bloch spaces) there are interesting subspaces and subsets of $\mathscr{L}$ for which many interesting things are known. Further the interested reader will find most of what is necessary for univalent functions in the books of Pommerenke [10], and Duren [5], and facts about the classical Hardy spaces in books by Garnett [6] or Cima, Matheson,and Ross [3]. Further, set

$$
\mathscr{L}_{1} \equiv\left\{f \in \mathscr{L}\left|\|f\|_{\mathscr{L}}=\int_{\mathbb{T}}\right| \log \left|f^{\prime}\left(e^{\imath t}\right)\right| \mid d t\right\} .
$$

It is shown in Cima and Pfaltzgraff [4], that $\mathscr{L}_{1}$ is a real, separable Banach space ( a subset of the harmonic Hardy space, $h^{1}$ ). In his paper [8], Hornich defined a subspace of $\mathscr{L}_{1}$ by requiring the following

$$
\mathscr{H} \equiv\left\{f \in \mathscr{L}_{1}\left|\sup _{(|z|<1)}\right| \arg f^{\prime}(z) \mid<\infty\right\} .
$$

Hornich equipped this space with a norm

$$
\|f\|_{\mathscr{H}}=\sup \left\{\left(\left|\arg f^{\prime}(z)-\arg f^{\prime}(w)\right| \quad \mid z \in D ; w \in D\right)\right\} .
$$

Equipped with this norm $\mathscr{H}$ becomes a real, separable Banach space. Note that the set $S$ of all normalized univalent functions on $D$ is not a subset of $\mathscr{H}$. For example univalent functions mapping onto spiral-like domains are not in $\mathscr{H}$. But there are many well known and well researched subsets of univalent functions in $\mathscr{H}$, see Cima and Pfaltzgraff [4], Ali and Vasudevarao [1]. Without attempting to supply a comprehensive list of references we give an abbreviated set of references with the understanding that the interested reader will find most of the necessary background in the references in the papers and books that are listed.

As noted, $S \cap \mathscr{H}$ contains several sets of functions which are distinguished by their additional geometric properties. Among these are the class $\mathcal{C}$ of normalized univalent functions of $S$ mapping onto convex domains, the class $\mathcal{S}$ of functions in $S$ mapping 
onto starlike domains and the class $\mathcal{K}$ of close to convex functions in $S$ (that is functions $f \in S$ for which there exists a starlike function $g \in S$ for which

$$
\Re \frac{z f^{\prime}(z)}{g(z)}>0,(|z|<1) .
$$

However, $\mathscr{H} \backslash S$ contains several other sets of functions which are locally univalent but not necessarily univalent. As examples we mention the following which come from the interesting paper, Ali and Vasudervarao [1]. Let $\alpha>0$ and set

$$
G(\alpha)=\left\{f \in \mathcal{A} \mid \Re\left(1+\frac{z f^{\prime \prime}(z)}{f^{\prime}(z)}\right)<1+\frac{\alpha}{2}\right\}
$$

Functions in $G(\alpha)$ for $\alpha \leq 1$ are univalent $(G(\alpha) \subset(S))$ but for $\alpha>1$ the functions in $G(\alpha)$ need not be univalent. In particular in [1], it is shown that if $f \in \mathscr{H}$ with $\|f\|_{\mathscr{H}} \leq$ $\alpha \pi$ then $f \in G(\alpha)$. Other examples of non-univalent classes containing functions in $\mathscr{H}$ are given there and we refer the interested reader to that source for these other examples and to many useful background references.

Most of the work on this topic has been done by researchers interested in geometric function theory, and much of it involves such topics as topological properties (separability and non-separability), extreme points of certain classical subsets of $\mathscr{H}$, the inclusion of certain well known subsets of analytic functions in $\mathscr{H}$, and inclusion of $\mathscr{H}$ in some classical Banach spaces of analytic functions on the unit disc. The following can be found in Cima and Pfaltzgraff [4] and Ali and Vasudevarao [1]. The notation $H^{p}(D)$ denotes the classical Hardy space of the unit disc.

Theorem 2.1. The space $\mathscr{H}$ is a subset of $\bigcup_{0<p<\infty} H^{p}(D)$.

In Section 5 we will strengthen this result.

\section{The SPACE BMOA}

Now we wish to point out several pertinent properties of the space BMO and BMOA on the unit circle. An excellent source for material on these sets is the set of notes by Girela [7]. An $L^{2}(\mathbb{T}, d m)$ function $f$ on the unit circle $\mathbb{T}$ is said to have bounded mean oscillation (BMO) if there is a positive number $\mathrm{M}$ such that for each interval $I \subset \mathbb{T}$ set

$$
U(f, I)=\frac{1}{|I|} \int_{I}\left|f(\zeta)-f_{I}\right| d m(\zeta) \leq M
$$

where $\zeta=\exp ^{\imath \theta}, d m(\zeta)=d \theta / 2 \pi$ and $f_{I}=\frac{\int_{I} f(\zeta) d m(\zeta)}{|I|}$. The set of such functions $f$ for which $U=\sup _{I} U(f, I)<\infty$ with pointwise sum and scalar products is a complex Banach space with

$$
\|f\|_{b}=|f(0)|+U \text {. }
$$

The set can be made into a Banach space in several ways. The space BMOA is the subspace of BMO consisting of functions from the Hardy spaces $H^{2} \bigcap \mathrm{BMO}($ mathbbT). In addition we will be considering a subspace of BMOA for which $f(0)=0$. The real variable version of this space $\mathrm{BMO}(\mathbb{T})$ (or more generally $\mathrm{BMO}\left(\mathbb{R}^{n}\right)$ ) suitably defined) has an illustrious history, but as we focus on $\operatorname{BMOA}(\mathbb{T})$ we will not try to outline this history but again mention the classic notes of Girela where the case $\operatorname{BMO}(\mathbb{T})$ is treated in detail. Some of the other useful equivalent definitions for the space BMOA are as follows. Assume $a \in D$ and $\phi_{a}$ is an automorphism of the unit disc mapping $a$ to zero. For $p \in(0, \infty)$ and $f$ analytic on $D$ define

$$
\|f\|_{\mathrm{BMOA}_{p}} \equiv|f(0)|+\sup _{a \in D}\left\|f_{a}\right\|_{H^{p}},
$$

where $f_{a} \equiv f \circ \phi_{a}-f(a)$. 
Theorem 3.1. With the above notation the following are equivalent:

(a) $\|f\|_{\mathrm{BMOA}_{p}}<\infty$.

(b) The family $\{f \circ S-f(S(0)) \mid S \in \operatorname{Aut}(D)\}$ is a bounded set in $H^{p}$.

(c) The family $\left\{f_{a} \mid a \in D\right\}$ is a bounded set in $H^{p}$.

All of these give equivalent definitions for BMOA. The space BMOA is not separable in this norm and the closure of the analytic polynomials in this norm is a separable closed subspace, labeled VMOA, Both VMOA and BMOA play roles in duality situations for the Hardy space theory.

In the case of analytic functions, for some time there were open problems related to some linear functional analysis questions for the classical Hardy spaces. In particular one such question was the following. What are the topological duals of the classical Hardy spaces $H^{p}(\mathbb{T})$ ? It was possible to satisfactorily identify the duals for $1<p<\infty$, but the question for $p=1$ had a functional analysis solution to the question stated in terms of a coset space. Namely to characterize the dual of $H^{1}(\mathbb{T})$ one uses duality between $L^{1}(T)$ and $L^{\infty}$ and an annihilator. Fefferman's initial work on BMOA showed that the space of analytic functions on the unit disc with bounded mean oscillation was the correct function space dual.

There are several important ways to recognize whether or not an analytic function on the unit disc is in this space. It is known that if an analytic function has a bounded real part then it is in BMOA. Further, if $f(z)=U(z)+\imath \widetilde{U}(z) \in$ BMOA and $U \in L_{\infty}(D)$ then since $\|\widetilde{U}\|_{L^{2}(\mathbb{T})} \leq C|| U \|_{L^{\infty}(\mathbb{T})}$ we have $\|f\|_{b} \leq C\|U\|_{L^{\infty}}$. Further, if $\mathrm{P}$ denotes the Riesz transform on $L^{2}(\mathbb{T})$ then it is well known that $f \in$ BMOA if and only if there is $\psi \in L^{\infty}(T)$ for which $f(z)=P(\psi)(z)$.

For our purposes we need one important representation of BMOA functions. For U a bounded, harmonic function of the unit disc $\mathrm{D}$, let $\widetilde{U}$ be a suitably normalized harmonic conjugate.

Theorem 3.2. For $F$ in BMOA there exist two bounded harmonic functions $U$ and $V$ so that

$$
F(z)=(U(z)+\imath \widetilde{U}(z))+\imath(V(z)+\imath \widetilde{V}(z))=F_{1}(z)+\imath F_{2}(z) .
$$

(Again see \{Girela [7], Theorem 7.2\} .)

Note although BMOA has many equivalent norms, the following is known.

Theorem 3.3. If $F=F_{1}+\imath F_{2}$ with $F_{j}, j=1,2$ as above then for some absolute constant $C$,

$$
\|F\|_{b} \leq C \quad \inf \left\{\|U\|_{\infty}+\|V\|_{\infty}\right\}
$$

as $F_{1}$ and $F_{2}$ vary over all such decompositions.

\section{The SUBSpace $\mathscr{K}$}

So let us define the real subspace of BMOA that we are interested in. Let

$$
\mathscr{K}=\left\{F \in \mathrm{BMOA} \mid F(0)=0, \Im(F(z)) \in L^{\infty}(\mathbb{T}) .\right.
$$

Proposition 4.1. The set $\mathscr{K}$ is a real subspace of BMOA. $\mathscr{K}$ is not a closed subset of BMOA.

Before supplying the proof of Proposition 4.1 we want to define the linear mapping in question between $\mathscr{H}$ and $\mathscr{K}$. For $f \in \mathscr{H}$ define

$$
L(f)(z)=F(z)=\ln \left(f^{\prime}(z)\right)=\ln \left|f^{\prime}(z)\right|+\imath \arg f^{\prime}(z)=U(z)+\imath \widetilde{U}(z), \quad z \in D .
$$

Note that by our assumptions $L$ is well defined by the above comments and $F \in$ BMOA. (Since $\arg f^{\prime}(z) \in L^{\infty}(D)$ one has $F \in$ BMOA by the above.) Further, $L(f)(0)=$ $F(0)=0$, implying that $L(f) \in \mathscr{K}$. 
Note that

$$
L(f \oplus g)(z)=L(f)(z)+L(g)(z)
$$

and

$$
L(r \odot g)(z)=r L(g)(z),
$$

so that $L$ is a linear mapping. It is now clear that $L$ is bounded since

$$
\|L(f)\|_{b} \leq C \cdot \sup \left\{\left|\arg f^{\prime}(z)\right| \mid z \in D\right\} \leq 2 C \cdot\|f\|_{\mathscr{H}} .
$$

By the normalization it is obvious that $L$ is one to one and further setting

$$
f(z)=\int_{o}^{z} \exp (F(w)) d w
$$

that $L$ has a linear inverse. Note that $\ln (1-z) \in \mathscr{K}$ but it is not in VMOA so that $\mathscr{K} \backslash V M O A$ is not empty.

We can now proceed to the proof of Proposition 4.1.

Proof. It is obvious that $\mathscr{K}$ is a real normed subspace of BMOA.

We give an example to show that $\mathscr{K}$ is not closed in BMOA. Let $\Omega$ be the unbounded domain, symmetric in the $x$ and $y$ axis, containing 0 and 1 , bounded on the right by a smooth curve $\Gamma$. Assume that $2 \in \Gamma$ and as $z \in \Gamma, \Im(z)>0$ tends to $\infty$ the curve tends smoothly to the positive real $y$ axis and reflect to get the unbounded domain $\Omega$ in $\mathbb{C}$. Then take the Riemann mapping $\phi$ from the unit disc onto $\Omega, \phi(0)=0, \phi^{\prime}(0)=1$. It is well known that $\phi$ is in VMOA. Hence, there is a sequence of polynomials, say $p_{n}$, tending to $\phi$ in VMOA and hence, uniformly on compacta of $\mathrm{D}$. We can normalize the $p_{n}$ to get a new family of polynomials, say $q_{n}$, with $q_{n}(0)=0, q_{n}^{\prime}(0)=1$, and with $q_{n}$ tending to $\phi$ in BMOA. Thus, $\phi$ is in $\overline{\mathscr{K}}$ but is not in $\mathscr{K}$.

Let us state at this point another important fact about BMOA, known as the JohnNirenberg Theorem and for which we shall have a use. First, recall the equivalent norms for BMOA, and use the notation $\mathrm{BMOA}_{p}$ for them, $\infty>p>0$. Fix $p$ as above and $F \in \mathrm{BMOA}$, and write

$$
\|F\|_{b}^{p}=\sup _{z \in D} \int_{\mathbb{T}}|F-F(z)|^{p} P_{z} d s=\sup _{z \in D}\left(|F|^{p}(z)-|F(z)|^{p}\right)
$$

where $P_{z}$ is the Poisson kernel evaluated at $\mathrm{z}$. The John-Nirenberg inequality (see Knese [9]) can be stated as follows.

Theorem 4.2. For $F \in \mathrm{BMOA}$ and any $\epsilon<\frac{2}{\sqrt{2}\|F\|_{\mathrm{BMOA}_{2}}}$, we have

$$
\int_{\mathbb{T}} \exp ^{\epsilon|F-F(z)|} P_{z} d s<\frac{3}{\left(1-\frac{\epsilon \sqrt{e}|| F \|_{\mathrm{BMOA}_{2}}}{2}\right)^{3 / 2}}
$$

\section{IMPLiCATIONS}

Recall that if $f \in \mathscr{H}$ then $f$ is in some Hardy space, and if $f \in \mathscr{L}_{1}$ then $\int_{\mathbb{T}}|\log | f^{\prime}\left(\exp ^{\imath \theta}\right)|| d \theta<$ $\infty$. In general functions in Hardy spaces need not have derivatives which are well behaved. For example there are functions in the disc algebra (hence in all the Hardy spaces) for which the derivatives are not in the Nevanlinna class. So a question to consider is the following. If $f$ is in $\mathscr{L}_{1}$ is $f^{\prime}$ in some Hardy space? We have not been able to answer this in this form but using information gleaned from the relationship established by the map $L$ we can give an answer when $f$ is in the Hornich space.

Theorem 5.1. If $f \in \mathscr{H}$ then there is a $p>0$ for which $f^{\prime} \in H^{p}$. 
Proof. Assume $f \in \mathscr{H}$, and $L(f)=F \in$ BMOA. Apply the John-Nirenberg Theorem, with $z=0$, to obtain

$$
\begin{aligned}
\int_{\mathbb{T}} \exp ^{\epsilon\left|F\left(\exp ^{2 \theta}\right)\right|} d \theta / 2 \pi & =\int_{\mathbb{T}} \exp ^{\epsilon\left|\log f^{\prime}\left(\exp ^{2 \theta}\right)\right|} d \theta / 2 \pi \geq \int_{\mathbb{T}} \exp ^{\epsilon|\log | f^{\prime}\left(\exp ^{2 \theta}\right)} \mid d \theta / 2 \pi \\
& \geq \int_{\mathbb{T} \cap\left(\left|f^{\prime}\right| \geq 1\right)}\left|f^{\prime}\left(\exp ^{\imath \theta}\right)\right|^{\epsilon} d \theta / 2 \pi
\end{aligned}
$$

Hence, $f^{\prime} \in H^{\epsilon}$. It is now well known that if $\epsilon<1$ then $f \in H^{q}$, where $q=\frac{\epsilon}{(1-\epsilon)}$ and if $\epsilon>1$, then $f \in \Lambda_{\alpha}, \alpha=1-1 / \epsilon$. The space $\Lambda_{\alpha}$ is the Lipschitz space of order $\alpha$.

We wish to introduce another mathematical object that plays a major role in the BMOA theory and for which we think the pullback to the $\mathscr{H}$ space is of interest. Namely, given a positive measure $d \mu(z)$ on the unit disc, $\mu$ is called a Carleson measure if for $h>0,|\zeta|=1$ and $\Omega(\zeta, h)$ the Carleson box in $\mathrm{D}$,

$$
\Omega(\zeta, h)=(z \in D|1-h<| z|<1,| \arg (z-\zeta) \mid<h),
$$

one has

$$
\mu(\Omega(\zeta, h)) \leq C h .
$$

It is known that for $F \in \mathrm{BMOA}$ the measure $d \mu(z)=\left|F^{\prime}\right|^{2}(1-|z|) d x d y$ is a Carleson measure.

For our interests this supplies a straightforward proof of the following.

Theorem 5.2. For every function $f \in \mathscr{H}$ the measure

$$
d \nu(z)=\left|\frac{f^{\prime \prime}}{f^{\prime}}\right|^{2}\left(1-|z|^{2}\right) d x d y
$$

is a Carleson measure. Consequently, for any $p$ the map from $H^{p}$ into $L^{p}(d \nu)$ is bounded. That is for $g \in H^{p}$ there is a $C(p)$ for which

$$
\left(\int|g(z)|^{p} d \nu(z)\right)^{1 / p} \leq C(p)\|g\|_{H^{p}}
$$

In order to discuss a last point we need to make a few more definitions.

Definition 5.3. For $\lambda>0$ let $\mathscr{G}(\lambda)$ denote the set of functions $g$, analytic and non-zero in the unit disc, satisfying

$$
|\arg g(z)| \leq \lambda,
$$

and

$$
|g(0)|=1
$$

The second definition is for a space known as weak $H^{1}$.

Definition 5.4. Denote by $H_{w}^{1}$ the weak Hardy-type space of order one. It consists of those analytic functions $f$ on the unit disc which are in the Smirnov (Nevanlinna) class and for which

$$
\mid\left\{\exp \imath \theta \in \mathbb{T}|| f(\exp \imath \theta \mid>t\} \mid<\frac{b}{t}\right.
$$

The notation $|*|$ denotes Lebesgue measure of $*$ on the unit circle. That is, the distribution function of $f$ on the unit circle is controlled. The letter $b$ is a constant depending on $f$. The infimum over all such $b$ is known as the weak $H^{1}$ norm and is denoted by $b(f)$.

With this notation Baernstein and Brown [2] proved the following. 
Theorem 5.5. Assume $F$ is a function satisfying

$$
F(z)=\lambda \cdot\left(\frac{\mu-z}{\delta-z}\right) \cdot f(z)
$$

where $\lambda, \mu$, and $\delta$ are in the unit circle and $f \in \mathscr{G}(\nu)$, where $\nu<\pi / 2$. Further, assume $F$ satisfies

Then $F$ is in $H_{w}^{1}$.

$$
|F(z)| \leq 2 /(1-|z|)
$$

We wish to add to this result for functions from the Hornich space.

Theorem 5.6. Assume $f$ is in the Hornich space, $\mathscr{H}$, and satisfies $\left|\arg f^{\prime}(z)\right| \leq \nu \leq$ $\pi / 2$ and the function $F$ satisfies

$$
F(z)=\lambda \cdot\left(\frac{\mu-z}{\delta-z}\right) \cdot f^{\prime}(z)
$$

where as above $\mu$ and $\delta$ are in the unit circle. Then $\ln \left|F^{\prime}(z)\right|$ is in $L^{p}(\mathbb{T})$ for all $p<\infty$.

This follows immediately since $\ln F(z) \in$ BMOA.

\section{Questions Related to this material}

The following are questions that we have considered in writing this and have been unable to answer.

Q 1. What is the closure of $\mathscr{K}$ in the space of BMOA functions that vanish at the origin?

Q 2. Assuming the operator $L^{-1}$ has been extended to $\overline{\mathscr{K}}$ what is its range, relative to $\mathscr{L}$ ?

Q 3. Can the function $F$ occurring in the Baernstein and Brown theorem be shown to be in the space CT of Cauchy-Transforms of finite Borel measures on the unit circle? Recall that $\mathrm{CT} \subseteq H_{w}^{1}$.

\section{REFERENCES}

1. Md F. Ali and A. Vasudevarao. On Certain Families in the Hornich Space, Comput. Methods Funct. Theory 18 (2018) 643-659.

2. A.Baernstein and J.E.Brown. Integral means of derivatives of monotone slit mappings, Comment. Math. Helvetici 57 (1982) 331-348.

3. J.Cima, A.L.Matheson and W.T.Ross. The Cauchy Transform. Math. Surveys and Monographs, Vol. 125, 2006, American Mathematics Society.

4. J.Cima, and J.Pfaltzgraff. A Banach space of locally univalent functions, Michigan Math. J., 17, (1970) 321- 334.

5. P.L.Duren. Theory of $H^{p}$ spaces. Vol. 38 Pure and Applied Mathematics, Academic Press, 1970.

6. J.B.Garnett. Bounded Analytic Functions, Graduate Texts in Mathematics 236, Springer, 2007.

7. D.Girela. Analytic Functions of Bounded Mean Osciallation, in Complex Function Spaces (Mekrijarvi, 1999), pp. 61-170, Univ. Joensuu Dept. Math. Rep. Ser. No. 4(2001).

8. H.Hornich. Ein Banachraum analytischer Funktionen in Zusammenhang mit den schlichten Funktionen, Monatsh. Math. 73 ( 1969) 36-45.

9. G.Knese. Uchiyame's Lemma and the John - Nirenberg inequality, arXiv 1201.5354. 25 Jan. 2012.

10. Ch. Pommerenke. Univalent Functions, Vandenhoeck and Ruprecht, Gottingen, 1974.

11. S.Yamashita. Gelfer functions, integral means, bounded mean oscillation, and univalency, Trans. AMS 321 (1990) 245-259.

Joseph A. Cima received his M.A. and Ph.D degrees at Pennsylvania State University. He is an emeritus professor of mathematics at the University of North Carolina at Chapel Hill. 
He has served as Secretary of the American Mathematics Society. His interests are in Several Complex Variable Theory, Operator Theory and Harmonic Analysis.

Dept. of Mathematics, University of North Carolina, Chapel Hill, NC 27599-3250, USA

E-mail address: cima@email.unc.edu 